\title{
On the Semantics of Logic Programs with Preferences
}

\author{
Sergio Greco \\ DEIS, Università della Calabria \\ via P. Bucci, 87030 Rende - Italy \\ Irina Trubitsyna \\ DEIS, Università della Calabria \\ via P. Bucci, 87030 Rende - Italy \\ Ester Zumpano \\ DEIS, Università della Calabria \\ via P. Bucci, 87030 Rende - Italy
}

GRECO@DEIS.UNICAL.IT

IRINA@DEIS.UNICAL.IT

ZUMPANO@DEIS.UNICAL.IT

\begin{abstract}
This work is a contribution to prioritized reasoning in logic programming in the presence of preference relations involving atoms. The technique, providing a new interpretation for prioritized logic programs, is inspired by the semantics of Prioritized Logic Programming and enriched with the use of structural information of preference of Answer Set Optimization Programming. Specifically, the analysis of the logic program is carried out together with the analysis of preferences in order to determine the choice order and the sets of comparable models. The new semantics is compared with other approaches known in the literature and complexity analysis is also performed, showing that, with respect to other similar approaches previously proposed, the complexity of computing preferred stable models does not increase.
\end{abstract}

\section{Introduction}

The increased interest in preferences is witnessed by an extensive number of proposals and systems for preference handling (Grell, Konczak, \& Schaub, 2005; Van Nieuwenborgh \& Vermeir, 2003; Wakaki, Inoue, Sakama, \& Nitta, 2003, 2004). The literature distinguishes static from dynamic preferences. Static preferences are fixed at the time a theory is specified, i.e. they are "external" to the logic program, whereas dynamic preferences appear within the logic program and are determined "on the fly". The most common form of preference consists in specifying preference conditions among rules (Brewka, 1996; Brewka \& Eiter, 1999, 2000; Delgrande, Schaub, \& Tompits, 2000a, 2000b, 2003; Gelfond \& Son, 1997; Schauba \& Wang, 2001; Van Nieuwenborgh \& Vermeir, 2002, 2004; Wang, Zhou, \& Lin, 2000; Zhang \& Foo, 1997), whereas, some recent proposals admit the expression of preference relations among atoms (Brewka, Niemela, \& Truszczynski, 2003; Brewka, 2004; Sakama \& Inoue, 2000; Wakaki et al., 2003). More sophisticated forms of preferences also allow the specification of priorities between conjunctions (disjunctions) of literals (Brewka et al., 2003; Delgrande et al., 2000a; Sakama \& Inoue, 2000) and numerical penalties for suboptimal options (Brewka, 2004).

This work is a contribution to prioritized reasoning in logic programming in the presence of preference conditions involving atoms. In particular, priorities are applied by following the natural ordering defined by dependencies, as proposed in the Answer Set Optimiza- 
tion ( $\mathcal{A S O}$ ) semantics (Brewka et al., 2003), and the comparison strategy, proposed in the Preferred Stable Model (PSM) semantics (Sakama \& Inoue, 2000), is reviewed by also introducing the concept of comparable models. The next example describes the intuition at the basis of the proposed approach.

Example 1 The following prioritized program $\left\langle\mathcal{P}_{1}, \Phi_{1}\right\rangle$, inspired by a program presented by Brewka et al. (2003), describes different menus and the preferences among drinks and desserts:

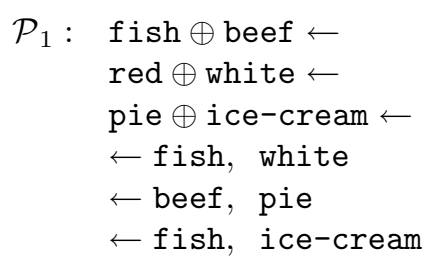

$\Phi_{1}: \varrho_{1}:$ white $>$ red $\leftarrow$ fish

$\varrho_{2}:$ red $>$ white $\leftarrow$ beef

$\varrho_{3}:$ pie $>$ ice-cream $\leftarrow$ red

The symbol $\oplus$ denotes exclusive disjunction, i.e. if the body of the rule is true exactly one atom in the head is true, whereas a rule with empty head defines a constraint, i.e. a rule which is satisfied only if the body is false. The first three rules of $\mathcal{P}_{1}$ select the main dish, the drink and the dessert; the last three rules are constraints and state that a feasible solution cannot contain i) fish and white or ii) beef and pie or iii) fish and ice-cream. Prioritized rules in $\Phi_{1}$ introduce preferences among drinks $\left(\varrho_{1}, \varrho_{2}\right)$ and desserts $\left(\varrho_{3}\right)$.

The program $\mathcal{P}_{1}$ has three stable models: $M_{1}=\{$ fish, red, pie $\}, M_{2}=$ beef, white, ice-cream $\}$ and $M_{3}=$ \{beef, red, ice-cream $\}$. The $\mathcal{P S M}$ returns $M_{1}$ as the unique preferred model; whereas the $\mathcal{A S O}$ technique, following the natural ordering of preference rules ( $\varrho_{1}$ and $\varrho_{2}$ precede $\varrho_{3}$ ), derives that $M_{3}$ is the unique solution. Thus, the two approaches provide different results.

The structure of preference rules in the above example suggests that i) fish and beef are alternative options for the main dish and ii) the choice of drink depends on the selected main dish and precedes the choice of dessert. The second conclusion is based on the observation that $\varrho_{1}$ and $\varrho_{2}$ provide opposite valuations of the choice of drink and they define two different classes of models (menus), which should be considered separately. In other words, the model $M_{1}$ (associated with the menu containing $\mathrm{fish}$ ) should not be compared with the models $M_{2}$ and $M_{3}$ (associated with the menus containing beef). Consequently, both $M_{1}$ and $M_{3}$ should be preferred.

Observe that in the above example the $\mathcal{P S M}$ semantics derives that $M_{1}$ is the preferred model as $M_{1}$ is preferable to $M_{3}$ (due to the presence of rule $\varrho_{3}$ ), $M_{3}$ is preferable to $M_{2}$ (due to the presence of rule $\varrho_{2}$ ) and, transitively, that $M_{1}$ is also preferable to $M_{2}$. It is worth noting that the use of the transitive closure makes the comparison of models much more complex as two models cannot be compared directly. On the other hand the $\mathcal{A S O}$ semantics is sensitive to syntactic changes of programs. This fact is illustrated by means of the following example.

Example 2 Consider the prioritized program $\left\langle P_{2}, \Phi_{2}\right\rangle$, an extension of the prioritized program defined in Example 1: 


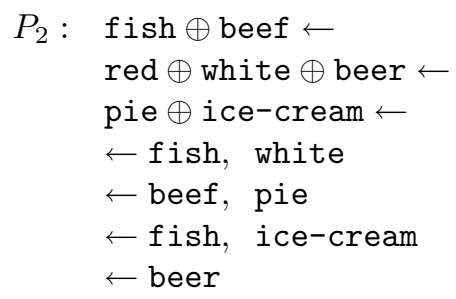

$\Phi_{2}: \widehat{\varrho}_{1}:$ beer $>$ white $>$ red $\leftarrow$ fish

$\widehat{\varrho}_{2}:$ beer $>$ red $>$ white $\leftarrow$ beef

$\widehat{\varrho}_{3}:$ pie $>$ ice-cream $\leftarrow$ red

This program is equivalent to the one reported in Example 1 as, even if it contains an additional choice (beer), this option is not feasible, for the presence of the constraint $\leftarrow$ beer. The set of stable models associated with the program $P_{2}$, coincides with those reported for the program $\mathcal{P}_{1}$ in Example 1, and consists of: $M_{1}=\{$ fish, red, pie $\}, M_{2}=$ \{beef, white, ice-cream $\}$ and $M_{3}=$ \{beef,red, ice-cream $\}$. Considering the set of preference rules, note that both preferences regarding the choice of drink have beer as the best option, but there is no stable model containing it. Intuitively, as the two problems $\left\langle\mathcal{P}_{1}, \Phi_{1}\right\rangle$ in Example 1 and $\left\langle P_{2}, \Phi_{2}\right\rangle$ are equivalent they must have the same preferred models. The $\mathcal{A S O}$ semantics is sensitive to the program change and gives $M_{1}$ and $M_{3}$ as a solution, whereas, for the equivalent program in Example 1, the returned preferred model is $M_{3}$ only. No change occurs in the set of preferred models for the $\mathcal{P S M}$ semantics.

Thus, in this paper we present a new semantics, inspired by the $\mathcal{P S M}$ and $\mathcal{A S O}$ semantics, which seems to better capture the intuitive meaning of programs and avoids the above mentioned problems.

\subsection{Contribution}

The paper provides a new semantics for prioritized logic programs enriching the one proposed by Sakama and Inoue (2000) with additional information gained from the structure of preference rules as proposed by Brewka et al. (2003). In particular, the new semantics uses different preference relations among stable models and introduces a natural ordering among preferences that fixes the order of choices, on the basis of the stratification of the preference program. Each decision is determined by the set of choices belonging to the corresponding level and provides the subset of models given in input as solution. Once a decision is made, this output subset becomes the input set of the following decision and so on. The proposed semantics drives the decision making process taking into account catching additional information regarding non comparable sets of models so partitioning the set of models of the program by looking at their alternative decisions. To this end the concept of incomparability, not taken into account by previous approaches, is introduced.

The paper presents a detailed comparison of the approaches dealing with preference relations among atoms. Particular attention is devoted to the $\mathcal{P S M}$ and the $\mathcal{A S O}$ semantics. The analysis of the complexity of computing preferred answer sets is also performed, showing that, w.r.t. previous proposals, such as the $\mathcal{P S M}$ and the $\mathcal{A S O}$ semantics, the complexity of computing preferred stable models does not increase. 


\subsection{Plan Of The Paper}

The rest of the paper is organized as follows: in Section 2 preliminaries on Disjunctive and Abductive Logic Programs, Prioritized Logic Programs and Answer Set Optimization semantics are given; in Section 3 a new interpretation for prioritized logic programs is presented; in Section 4 complexity results are provided; in Section 5 a comparison of the presented semantics with the $\mathcal{P S M}$ and $\mathcal{A S O}$ semantics is performed, and other approaches, known in the literature, are briefly described; finally, Section 6 outlines our conclusions.

\section{Preliminaries}

We assume familiarity with relational database theory, disjunctive logic programs, disjunctive deductive databases, (disjunctive) stable model semantics and computational complexity (Eiter, Gottlob, \& Mannila, 1997b; Gelfond \& Lifschitz, 1988, 1991; Papadimitriou, 1994).

\subsection{Background}

A (disjunctive) logic program is a finite set of rules $r$ of the form $a_{1} \vee \cdots \vee a_{k} \leftarrow b_{1}, \ldots, b_{m}$, not $c_{1}, \ldots$, not $c_{n}$ with $k+m+n>0$, where $a_{1}, \ldots, a_{k}, b_{1}, \ldots, b_{m}, c_{1}, \ldots, c_{n}$ are atoms. The disjunction $a_{1} \vee \cdots \vee a_{k}$, denoted by head $(r)$, is called head of $r$, while the conjunction $b_{1}, \ldots, b_{m}$, not $c_{1}, \ldots$, not $c_{n}$, denoted by $\operatorname{body}(r)$, is called body of $r$.

The Herbrand Universe $U_{\mathcal{P}}$ of a program $\mathcal{P}$ is the set of all constants appearing in $\mathcal{P}^{1}$, and its Herbrand Base $\mathcal{B}_{\mathcal{P}}$ is the set of all ground atoms constructed from the predicates appearing in $\mathcal{P}$ and the constants from $U_{\mathcal{P}}$. A term (resp. an atom, a rule or a program) is ground if no variables occur in it. A rule $r^{\prime}$ is a ground instance of a rule $r$, if $r^{\prime}$ is obtained from $r$ by replacing every variable in $r$ with some constant in $U_{\mathcal{P}} ; \operatorname{ground}(\mathcal{P})$ denotes the set of all ground instances of the rules in $\mathcal{P}$.

The intuitive meaning of the previous disjunctive rule is that if $\operatorname{body}(r)$ is true, i.e. $b_{1}, \ldots, b_{m}$ are true and $c_{1}, \ldots, c_{n}$ are false, then head $(r)$ must be true, i.e at least one of $a_{1}, \ldots, a_{k}$ has to be true (otherwise $r$ is not satisfied). Rules with empty head, called denials or constraints, will be used to define constraints and are satisfied only if the body is false. In this paper exclusive disjunction, denoted by $\oplus$, is used in the head; the statement head $(r)=$ $a_{1} \oplus \ldots \oplus a_{k}$ is true, if exactly one of $a_{1}, \ldots, a_{k}$ is true, i.e. a disjunctive rule of the form $a_{1} \oplus \cdots \oplus a_{k} \leftarrow$ body is a shorthand for the rule $a_{1} \vee \cdots \vee a_{k} \leftarrow$ body and $(k \times(k-1) / 2)$ constraints of the form $\leftarrow a_{i}, a_{j}$, body with $1 \leq i<j \leq k$. The solution of a logic program $\mathcal{P}$ is given in terms of stable model (answer set) semantics (Gelfond \& Lifschitz, 1988, 1991).

An interpretation $M$ for $\mathcal{P}$ is a model of $\mathcal{P}$ if $M$ satisfies all rules in $\operatorname{ground}(\mathcal{P})$. The minimal model semantics, defined for positive $\mathcal{P}$, assigns to $\mathcal{P}$ the set of its minimal models $\mathcal{M M}(\mathcal{P})$, where a model $M$ for $\mathcal{P}$ is minimal, if no proper subset of $M$ is a model for $\mathcal{P}$ (Minker, 1982). The more general disjunctive stable model semantics also applies to programs with (unstratified) negation (Gelfond \& Lifschitz, 1991). Disjunctive stable model semantics generalizes stable model semantics, previously defined for normal programs (Gelfond \& Lifschitz, 1988). For any interpretation $M$, denote with $\mathcal{P}^{M}$ the ground positive program derived from $\operatorname{ground}(\mathcal{P})$ by i) removing all rules that contain a negated atom

1. We are considering function free programs. 
not $a$ in the body and $a \in M$, and ii) removing all negated atoms from the remaining rules. An interpretation $M$ is a (disjunctive) stable model of $\mathcal{P}$ if and only if $M \in \mathcal{M M}\left(\mathcal{P}^{M}\right)$. For general $\mathcal{P}$, the stable model semantics assigns to $\mathcal{P}$ the set $\mathcal{S M}(\mathcal{P})$ of its stable models. It is well known that stable models are minimal models (i.e. $\mathcal{S M}(\mathcal{P}) \subseteq \mathcal{M M}(\mathcal{P})$ ) and that for negation free programs, minimal and stable model semantics coincide (i.e. $\mathcal{S M}(\mathcal{P})=\mathcal{M M}(\mathcal{P}))$.

\subsection{Extended And Abductive Programs}

Given an atom $p(t)$, a literal is either $p(t)$ or its strong negation $\neg p(t)$. An extended program is a program where atoms are replaced by literals. The semantics of extended disjunctive programs can also be given in terms of stable models by considering $p$ and $\neg p$ as different predicate symbols and considering the implicit constraint $\leftarrow p(X), \neg p(X)$ (Gelfond \& Lifschitz, 1991).

Abductive logic programming is an extension of logic programming to perform abductive reasoning (Kakas, Kowalski, \& Toni, 1992; Inoue \& Sakama, 1998). An abductive program (ALP) is a pair $\langle\mathcal{P}, \mathcal{A}\rangle$, where $\mathcal{P}$ is an extended program and $\mathcal{A}$ is a set of literals called abducibles. $\langle\mathcal{P}, \mathcal{A}\rangle$ can be represented by means of an extended program $\Gamma=\mathcal{P} \cup\{g(t) \vee$ $\left.g^{\prime}(t) \leftarrow \mid g(t) \in \mathcal{A}\right\} \cup\left\{g^{\prime}(t) \leftarrow \operatorname{not} g(t) \mid g(t) \in \mathcal{A}\right\}$.

Let $\Gamma$ be an ALP and $G$ a ground atom denoting an observation. Then, a set $S \subseteq \mathcal{A}$ is an explanation of $G$ iff there is a stable model $M$ of $\Gamma$ such that $S=M \cap \mathcal{A}$ and $G \in M$; a stable model $M$ is $\mathcal{A}$-minimal if there is no stable model $N$ such that $N \cap \mathcal{A} \subset \mathcal{M} \cap \mathcal{A}$. Moreover, $S$ is a minimal explanation (i.e. there is no explanation $S^{\prime} \subset S$ ) of $G$ iff $\Gamma \cup\{\leftarrow$ not $G\}$ has a consistent $\mathcal{A}$-minimal stable model $M$ such that $S=M \cap \mathcal{A}$ (Inoue \& Sakama, 1998).

It has been shown that given an ALP $\mathcal{P}$ and a ground atom $G$ denoting an observation, deciding whether there exists a $\mathcal{A}$-minimal explanation $S$ for $G$ is $\Sigma_{3}^{p}$-complete (Eiter, Gottlob, \& Leone, 1997a).

The rest of this section will briefly review the two main approaches for prioritizing reasoning we refer to, i.e. Prioritized Logic Programs and Answer Set Optimization, proposed, respectively, by Sakama and Inoue (2000) and Brewka et al. (2003).

\subsection{Prioritized Logic Programs}

A (partial) preference relation $\succeq$ among atoms is defined as follows: given two atoms $e_{1}$ and $e_{2}$, the statement $e_{1} \succeq e_{2}$ (called priority) means that $e_{1}$ has higher priority than $e_{2}$. Moreover, if $e_{1} \succeq e_{2}$ and $e_{2} \succeq e_{3}$, then $e_{1} \succeq e_{3}$. A priority statement $e_{1} \succeq e_{2}$ states that for each $a_{1}$ instance of $e_{1}$ and for each $a_{2}$ instance of $e_{2}$ the preference relation $a_{1} \succeq a_{2}$ holds. A prioritized logic program (PLP) is a pair $\langle\mathcal{P}, \Phi\rangle$ where $\mathcal{P}$ is a disjunctive program and $\Phi$ is a set of priorities. $\Phi^{*}$ denotes the set of priorities which can be reflexively or transitively derived from $\Phi$.

The statement $e_{1} \succ e_{2}$ stands for $e_{1} \succeq e_{2}$ and $e_{2} \nsucceq e_{1}$. Clearly, if $e_{1} \succ e_{2}$, the sets of ground instantiations of $e_{1}$ and $e_{2}$ have an empty intersection.

Definition 1 Given a prioritized logic program $\langle\mathcal{P}, \Phi\rangle$, the relation $\sqsupseteq$ is defined over the stable models of $\mathcal{P}$ as follows. For any stable models $M_{1}, M_{2}$ and $M_{3}$ of $\mathcal{P}$ : 
1. $M_{1} \sqsupseteq M_{1}$,

2. $M_{1} \sqsupseteq M_{2}$ if $\exists e_{1} \in M_{1}-M_{2}, \exists e_{2} \in M_{2}-M_{1}$ such that $\left(e_{1} \succeq e_{2}\right) \in \Phi^{*}$ and $\nexists e_{3} \in$ $M_{2}-M_{1}$ such that $\left(e_{3} \succ e_{1}\right) \in \Phi^{*}$,

3. if $M_{1} \sqsupseteq M_{2}$ and $M_{2} \sqsupseteq M_{3}$, then $M_{1} \sqsupseteq M_{3}$.

If $M_{1} \sqsupseteq M_{2}$ then $M_{1}$ is preferable to $M_{2}$. Moreover, if $M_{1} \sqsupseteq M_{2}$ and $M_{2} \sqsupseteq M_{1}$ then $M_{1} \sqsupset M_{2}$.

An interpretation $M$ is a preferred stable model of $\langle\mathcal{P}, \Phi\rangle$ if $M$ is a stable model of $\mathcal{P}$ and $N \sqsupseteq M$ implies $M \sqsupseteq N$ for any stable model $N$ (equivalently, if there is no interpretation $N$ which is a stable model of $\mathcal{P}$ and $N \sqsupset M)$. The set of preferred stable models of $\langle\mathcal{P}, \Phi\rangle$ will be denoted by $\mathcal{P S M}(\langle\mathcal{P}, \Phi\rangle)$. Note that the relation $\Phi_{1} \subseteq \Phi_{2}$ between two PLPs $\left\langle\mathcal{P}, \Phi_{1}\right\rangle$ and $\left\langle\mathcal{P}, \Phi_{2}\right\rangle$ does not imply $\mathcal{P S M}\left(\left\langle\mathcal{P}, \Phi_{2}\right\rangle\right) \subseteq \mathcal{P S M}\left(\left\langle\mathcal{P}, \Phi_{1}\right\rangle\right)$.

In PLP priority relations are used to express priorities over atoms, whereas priorities over more general forms of knowledge (conjunctive, disjunctive knowledge, rules, preconditions) can be expressed by a simple rewriting of the preference program. For instance, a preference rule with precondition of the form $\left(e_{1} \succeq e_{2}\right) \leftarrow B$ is expressed in PLP as $e_{1}^{\prime} \succeq e_{2}^{\prime}$, where $e_{1}^{\prime} \leftarrow e_{1}, B$ and $e_{2}^{\prime} \leftarrow e_{2}, B$.

The semantics of prioritized programs proposed by Sakama and Inoue (2000) will be denoted by $\mathcal{P S M}$ semantics. A sound and complete procedure that allows preferred answer sets for a PLP program to be computed using a generate and test algorithm has been proposed by Wakaki et al. (2003). This algorithm translates a PLP program $\langle\mathcal{P}, \Phi\rangle$ and any answer set $S$ of the program $\mathcal{P}$ into a single logic program $T[\mathcal{P}, \Phi, S]$, such that its answer sets are answer sets of $\mathcal{P}$ preferable to $S$. More details can be found in works presenting the semantics and the implementation (Sakama \& Inoue, 2000; Wakaki et al., 2004).

The complexity of answering queries over PLP programs is at least one level above the complexity of answering queries over standard programs (without preferences). In particular, let $\langle\mathcal{P}, \Phi\rangle$ be a prioritized logic program, then i) deciding the existence of a preferred stable model is $\Sigma_{2}^{p}$-hard; ii) deciding whether an atom is true in some (resp. all) preferable stable model of $\langle\mathcal{P}, \Phi\rangle$ is $\Sigma_{3}^{p}$-hard (resp. $\Pi_{3}^{p}$-hard). It is worth noting that in the original work (Sakama \& Inoue, 2000) it has been claimed that the complexity is exactly one level above the complexity of standard programs, but the proof does not take into account the transitivity property of the preference relation.

\subsection{Answer Set Optimization}

An answer set optimization program, denoted as ASO program, is a pair $\langle\mathcal{P}$, $\Phi\rangle$, where $\mathcal{P}$ is a disjunctive program, called Generating Program, and $\Phi$ is a Preference Program consisting of a finite set of rules of the form: $a_{1}>\cdots>a_{k} \leftarrow b_{1}, \ldots, b_{m}$, not $c_{1}, \ldots$, not $c_{n}$ where $b_{i} \mathrm{~s}$ and $c_{j} \mathrm{~s}$ are literals and $a_{i}$ s are boolean combinations ${ }^{2}$ of literals; here a literal is either an atom $L$ or its (strong) negation $\neg L$. $\Phi$ determines a preference ordering on the answer sets described by the generating program $\mathcal{P}$.

2. A boolean combination is a formula built of atoms by means of disjunctions, conjunctions, and default negation. 
Definition 2 Let $\langle\mathcal{P}, \Phi\rangle$ be an ASO program where $\Phi=\left\{\varrho_{1}, \ldots, \varrho_{n}\right\}$ and $S$ be an answer set of $\mathcal{P}$, then $S$ induces a satisfaction vector $V_{S}=\left(v_{S}\left(\varrho_{1}\right), \ldots, v_{S}\left(\varrho_{n}\right)\right)$ where:

- $v_{S}\left(\varrho_{j}\right)=I$, if $\varrho_{j}$ is Irrelevant to $S$, i.e. i) the body of $\varrho_{j}$ is not satisfied in $S$ or ii) the body of $\varrho_{j}$ is satisfied, but none of the atoms in the head of $\varrho_{j}$ is satisfied in $S$.

- $v_{S}\left(\varrho_{j}\right)=\min \left\{i|S|=a_{i} \wedge \varrho_{j}=a_{1}>\cdots>a_{k} \leftarrow b_{1}, . ., b_{m}\right.$, not $c_{1}, . .$, not $\left.c_{n}\right\}$, otherwise.

In the comparison of models it is assumed that $I$ is equal to 1 (i.e., $v_{S}\left(\varrho_{j}\right)=I$ is equivalent to $\left.v_{S}\left(\varrho_{j}\right)=1\right)$.

Definition 3 Let $S_{1}$ and $S_{2}$ be two answer sets, then i) $V_{S_{1}} \leq V_{S_{2}}$ if $v_{S_{1}}\left(\varrho_{i}\right) \leq v_{S_{2}}\left(\varrho_{i}\right)$ for every $i \in[1 . . n]$; ii) $V_{S_{1}}<V_{S_{2}}$ if $V_{S_{1}} \leq V_{S_{2}}$ and for some $i \in[1 . . n] v_{S_{1}}\left(\varrho_{i}\right)<v_{S_{2}}\left(\varrho_{i}\right)$. In these cases $S_{1} \sqsupseteq S_{2}$ and $S_{1} \sqsupset S_{2}{ }^{3}$, respectively.

A set of literals $S$ is an optimal model of an ASO program $\langle\mathcal{P}, \Phi\rangle$ if $S$ is an answer set of $\mathcal{P}$ and there is no answer set $S^{\prime}$ of $\mathcal{P}$ such that $S^{\prime} \sqsupset S$.

The complexity of ASO programs depends on the class of generating programs. For disjunctive programs the complexity of answering queries over ASO programs is exactly one level above the complexity of answering queries over standard programs (without preferences), i.e. i) deciding the existence of a preferred stable model is $\Sigma_{2}^{p}$-complete; ii) deciding whether a literal is true in some (resp. all) preferable stable model of $\langle\mathcal{P}, \Phi\rangle$ is $\Sigma_{3}^{p}$-complete (resp. $\Pi_{3}^{p}$-complete).

The strategy is further extended by introducing meta-preferences among preference rules: a ranked ASO program is a sequence $\left\langle\mathcal{P}, \Phi_{1}, \ldots, \Phi_{n}\right\rangle$ consisting of a generating program $\mathcal{P}$ and a sequence of pairwise disjoint preference programs $\Phi_{i}$. The rank of a rule $\varrho \in$ $\Phi_{1} \cup \cdots \cup \Phi_{n}$, denoted $\operatorname{rank}(\varrho)$, is the unique integer $i$ for which $\varrho \in \Phi_{i}$. Given two answer sets $S_{1}$ and $S_{2}, S_{1} \sqsupseteq_{\text {rank }} S_{2}$ if for every preference rule $\varrho^{\prime}$ such that $v_{S_{1}}\left(\varrho^{\prime}\right) \leq v_{S_{2}}\left(\varrho^{\prime}\right)$ does not hold, there is a rule $\varrho^{\prime \prime}$ such that $\operatorname{rank}\left(\varrho^{\prime \prime}\right)<\operatorname{rank}\left(\varrho^{\prime}\right)$ and $v_{S_{1}}\left(\varrho^{\prime \prime}\right)<v_{S_{2}}\left(\varrho^{\prime \prime}\right)$.

Moreover, a procedure deriving the natural ordering of the preference rules is introduced. Firstly, given a preference program $\Phi$, its dependency graph $G(\Phi)$ is defined. The atoms appearing in $\Phi$ form the vertex set of $G(\Phi)$. There is a directed edge from a vertex $b$ to a vertex $a$ in $G(\Phi)$ if there is a rule $\varrho$ in $\Phi$ such that $a$ appears in the head of $r$ and $b$ appears in the body of $r$. If the graph $G(\Phi)$ is acyclic, there is a natural ranking of its atoms which can be defined recursively as follows: $\operatorname{rank}(a)=0$ for every atom $a$ that has no predecessors in $G(\Phi)$; otherwise $\operatorname{rank}(a)$ is the maximum of the ranks of all predecessors of $a$ in $G(\Phi)$ incremented by 1 . The rank of a preference rule $\varrho$ is then defined as the maximum rank of atoms in its head.

The standard semantics of ASO programs, where priorities are examined all together, will be denoted as $\mathcal{A S O}$ semantics. The alternative semantics, where priorities are divided into strata following the natural order, will be denoted by $\mathcal{R} \mathcal{A S O}$ (ranked $\mathcal{A S O}$ ) semantics.

3. In the original work (Brewka et al., 2003) the symbols $\geq$ and $>$ are used instead of $\sqsupseteq$ and $\sqsupset$. 
(R)ASO semantics states that $M_{i} \sqsupseteq M_{2} \sqsupseteq M_{3}, M_{i} \sqsupseteq M_{6}$ and $M_{i} \sqsupseteq M_{4}$ for $i \in\{1,5\}$, i.e. in the $(\mathcal{R}) \mathcal{A S O}$ semantics the models $M_{1}$ and $M_{6}$ are compared directly, whereas in the $\mathcal{P S M}$ semantics the models $M_{1}$ and $M_{6}$ are compared transitively.

Before presenting the formal semantics of programs, some preliminary definitions are needed. A preference rule of the form $a_{1}>a_{2}>\cdots>a_{k} \leftarrow$ body is shorthand for the $k-1$ binary rules of the form $a_{i}>a_{i+1} \leftarrow$ body, with $i \in[1 . . k-1]$ and the set of preferences established by $\Phi$ is given by its transitive closure $\Phi^{*}$ defined as follows:

Definition 5 Given a prioritized program $\langle\mathcal{P}, \Phi\rangle$, the (ground) transitive closure of $\Phi$ is $\Phi^{*}=\Phi^{\prime} \cup\left\{a>c \leftarrow\right.$ body $y_{1}$, body $2 \mid a>b \leftarrow$ body $y_{1} \in \Phi^{*} \wedge b>c \leftarrow$ body $\left.y_{2} \in \Phi^{*} \wedge a \neq c\right\}$, where $\Phi^{\prime}$ is the set of binary preference rules derived from ground $(\Phi)$.

Thus, $\Phi^{*}$ is defined as the set of rules explicitly representing the preference relations among choice options. In Section 4 we will show that any ground prioritized program $\Pi=\langle\mathcal{P}, \Phi\rangle$ can be rewritten into an 'equivalent' program $\hat{\Pi}=\langle\hat{\mathcal{P}}, \hat{\Phi}\rangle$ such that $\hat{\Phi}^{*}$ contains a number of rules which is polynomial in the size of $\Pi$.

The structure of prioritized programs can be examined in order to establish the precedence relation among choices made. For instance, the presence of the preference rule $a>c \leftarrow b$ suggests that the selection of $b$ precedes the choice between $a$ and $c$ and thus establishes the precedence relation between $\{b\}$ and $\{a, c\}$. This idea was used by Brewka et al. (2003) for determining the natural ordering among preference rules. In more detail, the relational order among atoms appearing in $\Phi$ was captured by means of the corresponding dependency graph $G(\Phi)$; and the stratification of preference rules was established by considering their head atoms.

Unfortunately, the natural ordering among the preference rules can be established only if the corresponding dependency graph is acyclic. Thus, the presence of two rules coffee $>$ tea $\leftarrow$ pie and pie $>$ ice-cream $\leftarrow$ coffee in $\Phi$ does not admit the stratification of $\Phi$, as they introduce two "mutually dependent" choices.

The stratification algorithm proposed in this paper overcomes this problem by introducing the concept of "collapsed graph", which maps to the same node the options of "mutually dependent" choices.

Given a (ground) prioritized program $\Pi=\langle\mathcal{P}, \Phi\rangle, G_{\Pi}=(V, E)$ denotes the dependency graph whose set of nodes consists of all atoms in $\mathcal{P} \cup \Phi$ whereas there is an arc from $b$ to $a$ labeled $\phi($ resp. $p)$ if there is a rule in $\Phi($ resp. $\mathcal{P}$ ) containing $a$ in the head and $b$ in the body (resp. $b$ in either the head or the body). As the body of (preference) facts is empty, we assume that their bodies contain the built-in atom true, so that every fact $a \leftarrow$ can be considered as a rule $a \leftarrow$ true. $G_{\Pi}^{C}$ denotes the (acyclic) collapsed dependency graph derived from $G_{\Pi}$ by replacing maximal sets of mutual dependent nodes (i.e. nodes belonging to the same cycle) with a unique node. Clearly, each node in $G_{\Pi}^{C}$ is associated with a set of nodes in $G_{\Pi}$.

To each node in $G_{\Pi}^{C}$ it is possible to assign a level as follows:

- For each node $A$ in $G_{\Pi}^{C}$ with input degree zero, $\operatorname{level}(A)=0$; 
- For each node $A$ in $G_{\Pi}^{C}$ with input degree greater than zero, $\operatorname{level}(A)=\max \left\{\max \left\{\operatorname{level}(B)+1 \mid \exists(B, Z, \phi)\right.\right.$ in $\left.G_{\Pi}^{C}\right\}, \max \left\{\operatorname{level}(B) \mid \exists(B, Z, p)\right.$ in $\left.\left.G_{\Pi}^{C}\right\}\right\}$.

Observe that the function level assigns to each node the maximum distance from some node with input degree zero. The following definition introduces the concept of stratification for preference rules.

Definition 6 Stratification. Given a (ground) prioritized program $\Pi=\langle\mathcal{P}, \Phi\rangle, \Phi^{*}$ can be partitioned into $\left\langle\Phi^{*}[0], \Phi^{*}[1], \ldots, \Phi^{*}[n]\right\rangle$ subprograms (called strata) such that

- For each atom $a$ in $\Pi, \operatorname{level}(a)=\operatorname{level}(A)$, where $A$ is the node of $G_{\Pi}^{C}$ associated with $a$;

- For each rule $\varrho$ in $\Phi^{*}, \operatorname{level}(\varrho)=\max \{\operatorname{level}(a) \mid a \in \operatorname{Body}(\varrho)\}$;

- $\Phi^{*}[i]=\left\{\varrho \mid \varrho \in \Phi^{*} \wedge \operatorname{level}(\varrho)=i\right\}$ consists of all ground preference rules associated with the level $i$.

The partition of $\Phi^{*}$ into $\left\langle\Phi^{*}[0], \Phi^{*}[1], \ldots, \Phi^{*}[n]\right\rangle$ is called stratification.

The above definition of stratification of preference rules establishes the order in which preferences are applied by considering both $\mathcal{P}$ and $\Phi$. Moreover, the assignment of the level to each rule differs from the one proposed by Brewka et al. (2003) in two main aspects: the level of atoms is defined by analyzing the collapsed dependency graph and the level of rules is established by considering body atoms instead of head atoms. A more detailed comparison of the two approaches will be presented in Section 5 .

Example 4 Consider the prioritized program $\left\langle\mathcal{P}_{3}, \Phi_{3}\right\rangle$ of Example 3. The transitive closure $\Phi_{3}^{*}$ consists of the binary preference rules $\varrho_{1,1}:$ white $>$ red $\leftarrow$ fish, $\varrho_{1,2}:$ red $>$ beer $\leftarrow$ fish and $\varrho_{1,3}:$ white $>$ beer $\leftarrow$ fish, derived from $\varrho_{1}$, and the rules $\varrho_{2}, \varrho_{3}$ and $\varrho_{4}$. $\Phi_{3}^{*}$ can be stratified into the two strata $\Phi_{3}^{*}[1]=\left\{\varrho_{1,1}, \varrho_{1,2}, \varrho_{1,3}, \varrho_{2}, \varrho_{3}\right\}$ and $\Phi_{3}^{*}[2]=\left\{\varrho_{4}\right\}$.

The structural analysis performed in our approach goes beyond the stratification process and tries to understand the comparability of models. To this end the concepts of conflicting preferences and comparable models are introduced.

Two ground (binary) preferences of the form $a>b \leftarrow \operatorname{bod} y_{1}$ and $b>a \leftarrow \operatorname{bod} y_{2}$ are said to be conflicting. For instance, the preferences $\varrho_{1}$ : white $>$ red $\leftarrow$ fish and $\varrho_{2}:$ red $>$ white $\leftarrow$ beef of Example 1 are conflicting, whereas the preferences $\varrho_{1}$ and $\varrho_{2}^{\prime}$ : red $>$ water $\leftarrow$ beef are not. A set of preferences $\Phi$ is said to be conflicting if $\Phi^{*}$ contains two conflicting preference rules.

The intuition on the basis of our approach is clarified in this example. Suppose there are two conflicting preferences $\varrho_{1}: a>b \leftarrow c$ and $\varrho_{2}: b>a \leftarrow d$. The two conflicting preferences $\varrho_{1}$ and $\varrho_{2}$ specify the preference between $a$ and $b$ in two different sets of models, having $a$ or $b$, characterized by the presence of $c$ and $d$, respectively. Thus, $c$ and $d$, (and atoms on which $c$ and $d$ depend) define the alternative decisions. Once a decision has been made, the associated solutions (models) are no longer comparable.

A preference rule $\varrho$ is said to be relevant for a stable model $M$, if $\varrho$ can be used to compare $M$ with other stable models, that is if $M \models \operatorname{body}(\rho)$ and some of its head 
atoms belongs to $M$. Given a prioritized program $\langle\mathcal{P}, \Phi\rangle$ and a (ground) preference rule $\varrho: a>b \leftarrow \operatorname{bod} y \in \Phi^{*}$, the set of stable models, for which $\varrho$ is relevant, is $\mathcal{S M}(\mathcal{P}, \varrho)=$ $\{M|M \in \mathcal{S M}(\mathcal{P}) \wedge M| \operatorname{bod} y \wedge(a \vee b)\}$.

Definition 7 Comparable models. Let $\langle\mathcal{P}, \Phi\rangle$ be a prioritized program, $M_{1}$ and $M_{2}$ two stable models for $\mathcal{P}$ and $\left\langle\Phi^{*}[0], \ldots, \Phi^{*}[n]\right\rangle$ be a stratification of $\Phi^{*}$, then

1. $M_{1}$ and $M_{2}$ are comparable on $\Phi^{*}[0]$;

2. $M_{1}$ and $M_{2}$ are comparable on $\Phi^{*}[i+1]$ for $i \in[1 . . n]$, if

(a) they are comparable on $\Phi^{*}[i]$, and

(b) there do not exist two conflicting preference rules $\varrho_{1}, \varrho_{2} \in \Phi^{*}[i]$ such that $M_{1} \in \mathcal{S M}\left(\mathcal{P}, \rho_{1}\right)-\mathcal{S M}\left(\mathcal{P}, \rho_{2}\right)$ and $M_{2} \in \mathcal{S M}\left(\mathcal{P}, \rho_{2}\right)-\mathcal{S M}\left(\mathcal{P}, \rho_{1}\right)$.

Observe that, the second condition in the previous definition of comparable models states that the presence of two conflicting preference rules in a given level $i$ identifies two sets of models, for which only one of the two conflicting rules is relevant. Two models, appearing in the different sets have to be considered separately in the next levels. In other words, two stable models $M_{1}$ and $M_{2}$, having as relevant preferences the conflicting rules $\varrho_{1}: a>b \leftarrow \operatorname{bod} y_{1}$ and $\varrho_{2}: b>a \leftarrow \operatorname{bod} y_{2}$ in a given level $i$, are not comparable at levels greater than $i$, if $M_{1} \in \mathcal{S M}\left(\mathcal{P}, \rho_{1}\right)-\mathcal{S M}\left(\mathcal{P}, \rho_{2}\right)$ and $M_{2} \in \mathcal{S M}\left(\mathcal{P}, \rho_{2}\right)-\mathcal{S M}\left(\mathcal{P}, \rho_{1}\right)$, that is $M_{1} \models \operatorname{bod}_{1} \wedge(a \vee b) \wedge$ not body2 and $M_{2} \models$ body $_{2} \wedge(a \vee b) \wedge$ not body .

Example 5 Consider the stable models $M_{3}=$ ffish, beer,pie\}, $M_{6}=$ \{beef, beer, ice-cream $\}$, and the set of preference rules $\Phi_{3}^{*}$ of Example 4. The stable models $M_{3}$ and $M_{6}$ are comparable on $\Phi_{3}^{*}[0]$ by definition, while they are not comparable on $\Phi_{3}^{*}[1]$, because $\varrho_{1,3}$ is relevant only for $M_{3}$ (as $M_{3} \models$ fish $\wedge$ (white $\vee$ beer $) \wedge$ not beef), $\varrho_{3}$ is relevant only for $M_{6}$ (as $M_{6} \models$ beef $\wedge$ (white $\vee$ beer $) \wedge$ not fish), and these conflicting preferences belong to $\Phi_{3}^{*}[0]$.

Fact 1 Let $\langle P, \Phi\rangle$ be a prioritized program without conflicting preferences and $\left\langle\Phi^{*}[0]\right.$, $\left.\Phi^{*}[1], \ldots, \Phi^{*}[n]\right\rangle$ be the stratification of $\Phi^{*}$. Then, any two models $M_{1}$ and $M_{2}$ are comparable on $\Phi^{*}[i], i \in[0 . . n]$.

Proof. The proof of the above fact follows directly from Definition 7.

On the basis of Definition 7 the declarative semantics of prioritized logic programs can now be provided. This new semantics, denoted with $\mathcal{P A S}$ (Preferred Answer Sets), is given by preferred stable models as follows:

Definition 8 Preference between Answer Sets. Given a prioritized program $\langle\mathcal{P}, \Phi\rangle$, the relation $\sqsupseteq$ is defined over the stable models of $\mathcal{P}$ as follows. For any pair of stable models $M_{1}$ and $M_{2}$ of $\mathcal{P}$, being $\left\langle\Phi^{*}[0], \Phi^{*}[1], \ldots, \Phi^{*}[n]\right\rangle$ be the stratification of $\Phi^{*}, M_{1} \sqsupseteq M_{2}$ if

1. $\exists \varrho_{1}:\left(e_{1}>e_{2}\right) \leftarrow \operatorname{bod} y_{1} \in \Phi^{*}[i]$ such that $e_{1} \in M_{1}-M_{2}, e_{2} \in M_{2}-M_{1}$, such that $M_{1}$ and $M_{2}$ are comparable on $\Phi^{*}[i]$, and $\varrho_{1}$ is relevant for $M_{1}$ and $M_{2}$, and 
2. $\nexists \varrho_{2}:\left(e_{3}>e_{4}\right) \leftarrow \operatorname{bod} y_{2} \in \Phi^{*}[j]$, such that $j<i, e_{3} \in M_{2}-M_{1}, e_{4} \in M_{1}-M_{2}$ and $\varrho_{2}$ is relevant for $M_{1}$ and $M_{2}$.

Moreover, $M_{1}$ is strictly preferable to $M_{2}\left(M_{1} \sqsupset M_{2}\right)$ if $M_{1} \sqsupseteq M_{2}$ and $M_{2} \nsupseteq M_{1}$.

Note also that the relation $\sqsupset$ could be defined directly by replacing the condition $j<i$ with $j \leq i$ in Item 2 of the above definition.

Definition 9 Preferred Answer Sets. An interpretation $M$ is a preferred stable model for a prioritized program $\langle\mathcal{P}, \Phi\rangle$ if $M$ is a stable model of $\mathcal{P}$ and there does not exist a stable model $N$ such that $N \sqsupset M$. The set of preferred stable models for $\langle\mathcal{P}, \Phi\rangle$ will be denoted by $\mathcal{P} \mathcal{A S}(\langle\mathcal{P}, \Phi\rangle)$.

Note that Definition 8 introduces preferences between pairs of models by also considering additional information gained from the structure of preference rules.

Example 6 Consider the prioritized program $\left\langle P_{3}, \Phi_{3}\right\rangle$ of Example 3 and the stratification $\left\langle\Phi_{3}^{*}[0], \Phi_{3}^{*}[1]\right\rangle$ presented in Example 4 . We have that

- all models are comparable on $\Phi^{*}[0]$ by definition and

- owing to $\varrho_{1,1}, \varrho_{1,2}, \varrho_{1,3}, M_{1} \sqsupset M_{2} \sqsupset M_{3} ;$

- owing to $\varrho_{2}, \varrho_{3}, M_{5} \sqsupset M_{4}$ and $M_{6} \sqsupset M_{4}$;

- as $\varrho_{1,3}$ and $\varrho_{3}$ are conflicting, models $M_{1}$ and $M_{3}$, for which $\varrho_{1,3}$ is relevant, cannot be compared in $\Phi^{*}[1]$ with models $M_{4}$ and $M_{6}$, for which $\varrho_{3}$ is relevant.

Therefore, as discussed in Example $5, M_{3}$ and $M_{6}$ are not comparable on $\Phi_{3}^{*}[1]$ and, consequently, the preferred models are: $M_{1}, M_{5}$ and $M_{6}$.

In the previous example the stable model $M_{6}$ is considered as good as $M_{5}$ because both have beef as main dish, the best choice of drink (red wine and beer, respectively) and the same dessert (ice-cream). Observe that both $\mathcal{A S O}$ and $\mathcal{P S M}$ semantics discard $M_{6}$. As already stated, the $\mathcal{A S O}$ semantics deduces that $M_{1}$ and $M_{5}$ are preferable to $M_{6}$ owing to $\varrho_{3}$, while the $\mathcal{P S M}$ semantics states that $M_{1}$ is preferable to $M_{3}$ and $M_{3}$ is preferable to $M_{6}$, owing to $\varrho_{1}, \varrho_{3}$.

Example 7 Let $\left\langle\mathcal{P}_{3}, \Phi_{3}\right\rangle$ be the program of Example 3, consider the program $\left\langle\mathcal{P}_{3}, \widehat{\Phi}_{3}\right\rangle$ where $\widehat{\Phi}_{3}$ is derived from $\Phi_{3}$ by replacing $\varrho_{4}$ with

$$
\varrho_{4}^{\prime}: \text { pie }>\text { ice-cream } \leftarrow
$$

The new ground preference program has the unique level $\widehat{\Phi}_{3}^{*}[0]=\left\{\varrho_{1,1}, \varrho_{1,2}, \varrho_{1,3}, \varrho_{2}, \varrho_{3}, \varrho_{4}^{\prime}\right\}$, where $\varrho_{1,1}, \varrho_{1,2}$ and $\varrho_{1,3}$ are derived from $\varrho_{1}$, as shown in Example 4. Due to $\varrho_{4}^{\prime}$, the following relations also hold: $M_{i} \sqsupset M_{j}$ for $i \in\{1,2,3\}$ and $j \in\{4,5,6\}$. Therefore, $M_{1}$ is the unique preferred model. The same result is obtained by both $\mathcal{P S M}$ and $\mathcal{A S O}$ semantics. 


\section{Complexity}

This section provides some results concerning the computational complexity of computing preferred stable models and answering queries under $\mathcal{P} \mathcal{A S}$ semantics. We consider here data complexity where the input domain $U_{\mathcal{P}}$ consists of the Herbrand universe (we assume that all constants occurring in $\Phi$ also occur in $\mathcal{P}$ ). Clearly, the size of the Herbrand base $\mathcal{B}_{\mathcal{P}}$ as well as the sizes of $\operatorname{ground}(\mathcal{P})$ and $\operatorname{ground}(\Phi)$ are polynomial in the size of $U_{\mathcal{P}}$.

The following results demonstrate that allowing preferences among atoms in the semantics proposed here increases the complexity and expressivity of the language by one level in the polynomial hierarchy. Thus the use of additional information does not increase the computational complexity of the proposed approach with respect to the above mentioned techniques (Brewka et al., 2003; Sakama \& Inoue, 2000).

Proposition 1 Let $\Pi=\langle\mathcal{P}, \Phi\rangle$ be a prioritized program, then there exists a program $\hat{\Pi}=$ $\langle\hat{\mathcal{P}}, \hat{\Phi}\rangle$ equivalent to $\left\langle\right.$ ground $\left.(\mathcal{P}), \Phi^{*}\right\rangle$ such that i) the stratification of $\hat{\Phi}$ can be computed in polynomial time, and ii) $\langle\hat{\mathcal{P}}, \hat{\Phi}\rangle$ can be derived from $\left\langle\operatorname{ground}(\mathcal{P}), \Phi^{*}\right\rangle$ in polynomial time.

Proof. We start by considering the program $\Pi^{\prime}=\left\langle\operatorname{ground}(\mathcal{P}), \Phi^{\prime}\right\rangle$, where $\Phi^{\prime}$ is the binary ground version of $\Phi$. The size of the graphs $G_{\Pi^{\prime}}$ and $G_{\Pi^{\prime}}^{C}$ is polynomial in the size of $\Pi^{\prime}$ and can be computed in polynomial time. As the assignment of levels to nodes in $G_{\Pi^{\prime}}^{C}$ can be done in polynomial time, the assignment of levels to atoms and rules in $\Phi^{\prime}$ can also be done in polynomial time.

Let $\left\langle\Phi^{\prime}[0], \ldots, \Phi^{\prime}[n]\right\rangle$ be the stratification of $\Phi^{\prime}$. We generate a new ground prioritized $\operatorname{program}\langle\hat{\mathcal{P}}, \hat{\Phi}\rangle$ which is equivalent to $\left\langle\operatorname{ground}(\mathcal{P}), \Phi^{*}\right\rangle$ and such that the size of $\hat{\Phi}$ is polynomial in the size of $\Phi^{\prime}$.

Initially, assign to each ground atom $a_{i}$ appearing in the head of a preference rule in $\Phi^{\prime}$ a unique index $i$. Let $a_{1}, \ldots, a_{p}$ be the (indexed) atoms appearing in the head of rules in $\Phi^{\prime}$, $\hat{\mathcal{P}}$ denotes the program $\operatorname{ground}(\mathcal{P}) \cup \mathcal{P}_{\Phi}$ where

$$
\begin{aligned}
\mathcal{P}_{\Phi}= & \left\{b(i, j, l) \leftarrow b o d y_{i, j} \mid a_{i}>a_{j} \leftarrow \operatorname{bod} y_{i, j} \in \Phi^{\prime}[l]\right\} \cup \\
& \left\{b(i, j, l) \leftarrow b\left(i, k, l_{1}\right), b\left(k, j, l_{2}\right), l=\max \left(l_{1}, l_{2}\right) \mid i, j, k \in[1 . . p], l_{1}, l_{2} \in[1 . . n]\right\}
\end{aligned}
$$

and $b$ is a new predicate symbol. Then, $\hat{\Phi}$ denotes the new set of ground preference rules defined as follows:

$$
\hat{\Phi}=\left\{a_{i}>a_{j} \leftarrow b(i, j, l) \mid i, j \in[1 . . p] \wedge l \in[0 . . n]\right\}
$$

The stratification of $\hat{\Phi}$ is obtained by associating to each stratum $l$ the preference rules whose body atom has the value of the level attribute equal to $l$, that is $\hat{\Phi}[l]=\left\{a_{i}>a_{j} \leftarrow\right.$ $\left.b(i, j, l) \mid a_{i}>a_{j} \leftarrow b(i, j, l) \in \hat{\Phi}\right\}$.

In order to show the equivalence between $\left\langle\operatorname{ground}(\mathcal{P}), \Phi^{*}\right\rangle$ and $\langle\hat{\mathcal{P}}, \hat{\Phi}\rangle$, observe that the set of stable models of $\operatorname{ground}(\mathcal{P})$ and $\hat{\mathcal{P}}$ are 'equivalent', i.e. for each $M \in \mathcal{S M}(\hat{\mathcal{P}})$ there is a stable model $N \in \mathcal{S M}(\operatorname{ground}(\mathcal{P}))$ such that $N=M-\{b(i, j, l) \mid b(i, j, l) \in M\}$ and for each $N \in \mathcal{S M}(\operatorname{ground}(\mathcal{P}))$ there is a stable model $M \in \mathcal{S M}(\hat{\mathcal{P}})$ such that $N=$ $M-\{b(i, j, l) \mid b(i, j, l) \in M\}$, as the rules in $\operatorname{ground}(\mathcal{P})$ do not contain atoms of the form $b(i, j, l)$ in their bodies. 
Moreover, let $N$ be a stable model of $\operatorname{ground}(\mathcal{P})$ and $M$ be the corresponding stable model of $\hat{\mathcal{P}}(N \subseteq M)$, for each ground preference rule $a_{i}>a_{j} \leftarrow$ body $y_{i, j}$ in $\Phi^{*}[l]$ whose body is true in $N$, there is a ground rule $a_{i}>a_{j} \leftarrow b(i, j, l)$ in $\hat{\Phi}[l]$ whose body is also true in $M$ and vice versa. Therefore the two sets $\hat{\Phi}[l]$ and $\Phi^{*}[l]$ are equivalent, for all $l \in[1 . . n]$.

Clearly, the program $\langle\hat{\mathcal{P}}, \hat{\Phi}\rangle$ is derived from $\left\langle\operatorname{ground}(\mathcal{P}), \Phi^{*}\right\rangle$ in polynomial time.

In the following, for the sake of simplicity of presentation, we continue to refer to the program $\langle\mathcal{P}, \Phi\rangle$ and to the stratification of $\Phi^{*}$.

Proposition 2 Let $\langle\mathcal{P}, \Phi\rangle$ be a prioritized program, $M_{1}$ and $M_{2}$ two stable models for $\mathcal{P}$, and $\left\langle\Phi^{*}[0], \Phi^{*}[1], \ldots, \Phi^{*}[n]\right\rangle$ a stratification of $\Phi^{*}$. The problem of checking whether, for a given $k \leq n, M_{1}$ and $M_{2}$ are comparable on $\Phi^{*}[0], \ldots, \Phi^{*}[k]$ can be solved in polynomial time.

Proof. Obviously $M_{1}$ and $M_{2}$ are comparable in $\Phi^{*}[0]$. Assuming that $M_{1}$ and $M_{2}$ are comparable for a given level $j<k, M_{1}$ and $M_{2}$ are comparable for the level $j+1$ if there are no two conflicting preference rules $\rho_{1}=a>b \leftarrow b o d y_{1}$ and $\rho_{2}=b>a \leftarrow b o d y_{2}$ in $\Phi^{*}[j]$ such that $M_{1}=\operatorname{bod} y_{1} \wedge(a \vee b) \wedge$ not body $y_{2}$ and $M_{2}=\operatorname{bod} y_{2} \wedge(a \vee b) \wedge$ not body . This check can be done in polynomial time as the number of rules in $\Phi^{*}[j]$ is polynomial in the size of $U_{\mathcal{P}}$. Moreover, as the maximum value of $k$ is bounded by the size of $\Phi^{*}$ (which is bounded by the set of atoms in $\mathcal{B}_{\mathcal{P}}$ ), the global complexity is also polynomial.

Corollary 1 Let $\langle\mathcal{P}, \Phi\rangle$ be a prioritized program, $M_{1}$ and $M_{2}$ two interpretations for $\mathcal{P}$. The problem of checking whether $M_{1} \sqsupset M_{2}$ can be solved in polynomial time.

Proof. Straightforward from Definition 8 and Proposition 2.

Lemma 1 Let $\langle\mathcal{P}, \Phi\rangle$ be a prioritized program and $M$ an interpretation for $\mathcal{P}$. The problem of deciding whether $M$ is a preferred stable model for $\langle\mathcal{P}, \Phi\rangle$ is in $\Pi_{2}^{p}$.

Proof. Consider the complementary problem of deciding whether $M$ is not a preferred stable model for $\langle\mathcal{P}, \Phi\rangle$. In such a case it is sufficient to first check if $M$ is a stable model. If $M$ is a stable model it is sufficient to guess an interpretation $N$ and to check that i) $\mathrm{N}$ is a stable model for $\mathcal{P}$ and ii) $N \sqsupset M$. The check on part i) (as well as the check that $M$ is a stable model) can be done by means of a $\mathcal{N P}$ oracle as the problem of deciding whether an interpretation is a stable model for a disjunctive program is $c o \mathcal{N} \mathcal{P}$-complete, whereas the check on part ii) can be done in polynomial time (see Corollary 1). Therefore, the complexity of the complementary problem is $\mathcal{N} \mathcal{P}^{\mathcal{N} \mathcal{P}}$ and, consequently, the complexity of the original problem is $c o \mathcal{N} \mathcal{P}^{\mathcal{N} \mathcal{P}}$.

Theorem 1 Let $\langle\mathcal{P}, \Phi\rangle$ be a prioritized program. Then

1. Deciding whether a ground atom $G$ is true in some preferred stable models of $\langle\mathcal{P}, \Phi\rangle$ is $\Sigma_{3}^{p}$-complete;

2. Deciding whether a ground atom $G$ is true in all preferred stable models of $\langle\mathcal{P}, \Phi\rangle$ is $\Pi_{3}^{p}$-complete. 
Proof. Membership: We first demonstrate that deciding whether $G$ is true in some preferred stable model of $\langle\mathcal{P}, \Phi\rangle$ is in $\Sigma_{3}^{p}$. This result suffices to prove that the complementary problem, consisting in deciding whether $A$ is true in all preferred stable models, is $\Pi_{3}^{p}$-complete.

To show the membership it is sufficient to guess an interpretation $M$ containing $G$ and to check whether $M$ is a preferred stable model. From Lemma 1 the problem of deciding whether $M$ is a preferred stable model is in $\Pi_{2}^{p}$ and can be solved by means of a $\Sigma_{2}^{p}$ oracle. Therefore, deciding whether there exists a preferred stable model containing $G$ is in $\mathcal{N P}^{\Sigma_{2}^{p}}=\Sigma_{3}^{p}$.

Hardness: Given an abductive logic program consisting of a disjunctive program $\mathcal{P}$ and a set of abducibles (positive) atoms $\mathcal{A}$, the ground abductive logic program derived prom $\mathcal{P}$ and $\mathcal{A}$ is

$$
\begin{aligned}
\Gamma=\operatorname{ground}(\mathcal{P}) \cup\left\{g^{\prime}(t) \leftarrow \operatorname{not} g(t) \mid g(t) \in \operatorname{ground}(\mathcal{A})\right\} \\
\cup\left\{g^{\prime}(t) \oplus g(t) \leftarrow \quad \mid g(t) \in \operatorname{ground}(\mathcal{A})\right\}
\end{aligned}
$$

Let

$$
\Phi=\left\{g^{\prime}(t)>g(t) \leftarrow \mid g(t) \in \operatorname{ground}(\mathcal{A})\right\}
$$

$\langle\Gamma, \Phi\rangle$ denotes the prioritized program derived from $\mathcal{P}$ and $\mathcal{A}$.

For any two stable models $M, N \in \mathcal{S M}(\Gamma), M \sqsupseteq N$ with respect to $\Phi$ means that a preference $p^{\prime}(u)>p(u) \leftarrow \in \Phi$ such that $p^{\prime}(u) \in M$ and $p(u) \in N$ exists and no preference $q^{\prime}(v)>q(v) \leftarrow \in \Phi$ such that $q^{\prime}(v) \in N$ and $q(v) \in M$ exists. This implies that $M \cap \operatorname{ground}(\mathcal{A}) \subseteq N \cap \operatorname{ground}(\mathcal{A})$ and, consequently, that preferred stable models are $\mathcal{A}$-minimal.

Therefore, the problem of deciding whether $\langle\Gamma, \Phi\rangle$ has an $\mathcal{A}$-minimal explanation for a goal $G$ is equivalent to deciding whether $\langle\Gamma, \Phi\rangle$ has a preferred stable model containing $G$. Consequently, as the problem of deciding whether a $\mathcal{A}$-minimal explanation $S$ for $G$ exists is $\Sigma_{3}^{p}$-complete, the problem of deciding whether a preferred stable model $M$ for $\langle\Gamma, \Phi\rangle$ containing $G$ exits is also $\Sigma_{3}^{p}$-hard, whereas the problem of deciding whether all preferred stable models of $\langle\mathcal{P}, \Phi\rangle$ contain $G$ is $\Pi_{3}^{p}$-hard.

Corollary 2 Let $\langle\mathcal{P}, \Phi\rangle$ be a disjunction-free, prioritized program. Then deciding whether a ground atom is true in some (all) preferred stable models of $\langle\mathcal{P}, \Phi\rangle$ is $\Sigma_{2}^{p}$-complete $\left(\Pi_{2}^{p}\right.$-complete).

Proof. The complexity is one level lower as the problem of deciding whether an interpretation $M$ is a stable model for a disjunction-free program is polynomial.

\section{Analysis And Comparison}

This section compares the semantics introduced here with the $\mathcal{P S M}$ and (R)ASO semantics and briefly discusses other recently proposed semantics.

The $\mathcal{P S M}$ semantics is very elegant and compares pairs of models on the basis of their common preferences and not on the basis of their degree of satisfaction. It does not consider the natural ordering between preference rules and, in some cases, as in Example 1 and 3, compares (and consequently discards) models which in the $\mathcal{P} \mathcal{A S}$ approach are not 
comparable. An interesting feature of the $\mathcal{P S M}$ technique is the application of transitive property in order to derive additional preference relations among problem solutions so that new, not immediately visible, preference relations are captured. However, as the test of the transitive property cannot be performed by a direct comparison of two models, this lies in a more complex implementation.

The $(\mathcal{R}) \mathcal{A S O}$ technique is a very powerful tool as it determines the preferred models by evaluating the degree of satisfaction of all preference rules. Thus, it compares two models even in the absence of common preferences; and the preference relation between the two models can be established directly. In more detail, the $\mathcal{R} A \mathcal{S O}$ technique considers the structure of preference rules by associating a degree of satisfaction to choice options and introduces a natural ordering among preferences. As in the case of $\mathcal{P S M}$ semantics, the (R)ASO semantics also compares and, consequently, discards models which are not comparable using the $\mathcal{P} \mathcal{A S}$ technique. For instance, for the program $\left\langle P_{1}, \Phi_{1}\right\rangle$, presented in the Introduction, $\mathcal{R} A S O$ discards $M_{1}$, having the second best option of drink, even if this is the unique possible choice in the presence of $\mathrm{fish}$.

More specifically, the preference relation $\sqsupseteq$ used in the $\mathcal{P S M}$ approach is a preorder relation as it is reflexive and transitive; it determines equivalent answer set classes and establishes the partial preference order among the above mentioned classes. Consequently the preferred answer sets are those appearing in the preferred classes. It should be noted that $\mathcal{P S M}$ semantics requires the use of the transitive property in order to derive, on the basis of relations obtained by the direct comparison of pairs of models, new preference relations. On the contrary, the $(\mathcal{R}) \mathbf{A S O}$ semantics uses a strict preference relation which is just asymmetric and it does not require the application of the transitive property to compare solutions.

The $\mathcal{P A S}$ semantics, proposed here, compares two solutions on the basis of their common preferences by introducing the concept of comparable models and by considering a refinement of the natural order among preference choices. Thus, it can be seen as an extension of $\mathcal{P S M}$ semantics that also uses additional information derived from the structure of preference rules, but which instead of comparing models transitively, compares models by considering the transitive closure of the (ground) preference rules.

A novelty of $\mathcal{P} \mathcal{A S}$ semantics is the consideration of the structural information of preference rules. It introduces the concept of comparable models in order to avoid comparing models which (in our opinion) should not be compared as they are associated with alternative decisions. Moreover, it proposes a refinement of the natural order among preferences in order to define the order of choices. $\mathcal{R A S O}$ semantics establishes the relational order among atoms appearing in $\Phi$ by means of the corresponding dependency graph $G(\Phi)$ and cannot treat the case of "mutually dependent" choices. The stratification algorithm, proposed in this paper, overcomes this problem by considering the collapsed graph $G_{\Pi}^{C}$, which is acyclic by construction and is not sensitive to "syntactic" changes. Moreover, in $\mathcal{R} A \mathcal{A O}$ semantics the stratification of preference rules is established by considering their head atoms; whereas in $\mathcal{P} \mathcal{A S}$ semantics levels are assigned to rules on the basis of the body atoms, following the intuition that they describe the contexts of choices. Thus, the stratification proposed here always assigns preference facts to the first level because the level of a rule is fixed by looking at the level of body atoms.

Some of the advantages of the adopted approach are clarified by the following example. 
The program has four stable models: $M_{1}=$ ffish, white $\}, M_{2}=$ \{beef, red,$M_{3}=$ \{pork, beer $\}$ and $M_{4}=$ \{chicken, water\}, whereas the direct preference relations, for the $\mathcal{P S M}$ semantics, are as follows: $M_{1} \sqsupseteq M_{2}, M_{2} \sqsupseteq M_{1}, M_{3} \sqsupseteq M_{4}, M_{4} \sqsupseteq M_{3}$ and $M_{2} \sqsupseteq M_{4}$.

Consequently, we have that the graph $G_{\mathcal{P S M}}$ consists of four nodes $\left(M_{1}, M_{2}, M_{3}\right.$ and $M_{4}$ ) and four arcs: $M_{1} \sqsupset M_{3}, M_{1} \sqsupset M_{4}, M_{2} \sqsupset M_{3}$ and $M_{2} \sqsupset M_{4}$. Therefore, the preferred models are $M_{1}$ and $M_{2}$.

Regarding the $\mathcal{P} \mathcal{A S}$ semantics, we have that only the relation $M_{2} \sqsupset M_{4}$ holds and, thus, there are three preferred stable models, namely $M_{1}$ and $M_{2}$ and $M_{3}$.

Theorem 2 For any prioritized program $\langle\mathcal{P}, \Phi\rangle$ such that $\Phi$ consists in preference facts, $\mathcal{P S M}(\langle\mathcal{P}, \Phi\rangle) \subseteq \mathcal{P} \mathcal{A} \mathcal{S}(\langle\mathcal{P}, \Phi\rangle)$.

Proof. Consider the graphs $G_{\mathcal{P S M}}=\left(V, E_{\mathcal{P S M}}\right)$ and $G_{\mathcal{P A S}}=\left(V, E_{\mathcal{P A S}}\right)$. Both graphs are acyclic and $E_{\mathcal{P A S}} \subseteq E_{\mathcal{P S M}}$. As the two graphs are acyclic, by adding edges which do not create cycles, the number of nodes without incoming edges decreases. Therefore, the set of nodes without incoming edges in $G_{\mathcal{P A S}}$ contains all nodes without incoming edges in $G_{\mathcal{P S M}}$ and, consequently, $\mathcal{P S M}(\langle\mathcal{P}, \Phi\rangle) \subseteq \mathcal{P} \mathcal{A} \mathcal{S}(\langle\mathcal{P}, \Phi\rangle)$.

We now analyze the relation between $\mathcal{A S O}$ and $\mathcal{P A S}$ semantics. First of all note that, as observed in the Introduction, the $\mathcal{A S O}$ semantics is sensitive to syntax changes. For instance, the prioritized program

$$
\begin{array}{ll}
\mathrm{a} \oplus \mathrm{b} \oplus \mathrm{c} \leftarrow & \rho_{1}=\mathrm{a}>\mathrm{b}>\mathrm{c} \leftarrow \\
& \rho_{2}=\mathrm{b}>\mathrm{a} \leftarrow
\end{array}
$$

has two preferred stable models: $M_{1}=\{\mathrm{a}\}$ and $M_{2}=\{\mathrm{b}\}$. However, for the program below

$$
\begin{array}{ll}
\mathrm{a} \oplus \mathrm{b} \oplus \mathrm{c} \leftarrow \quad & \rho_{1}^{\prime}=\mathrm{a}>\mathrm{b} \leftarrow \\
& \rho_{1}^{\prime \prime}=\mathrm{b}>\mathrm{c} \leftarrow \\
& \rho_{2}=\mathrm{b}>\mathrm{a} \leftarrow
\end{array}
$$

derived from the rewriting of the rule $\rho_{1}, M_{3}=\{\mathrm{c}\}$ is also a preferred model.

Thus, we consider a special class of constraints which is not sensitive to "syntactic" changes. Since every (ground) prioritized program $\Pi=\langle\mathcal{P}, \Phi\rangle$, such that $\Phi^{*}$ can be partitioned into $n$ strata, with $n>1$, can be rewritten into a program $\hat{\Pi}=\langle\hat{\mathcal{P}}, \hat{\Phi}\rangle$, such that $\hat{\Pi}$ and $\Pi$ are equivalent under the $\mathcal{P} \mathcal{A} \mathcal{S}$ semantics (as shown in the proof of Proposition 1 ), and may not be equivalent under the $\mathcal{R} \mathcal{A S O}$ semantics (as all rules in $\hat{\Phi}$ belong to the unique stratum 0 ), we continue to consider programs $\Pi=\langle\mathcal{P}, \Phi\rangle$ where $\Phi^{*}$ consists of a single stratum and, in particular, of facts.

Given a prioritized program $\langle\mathcal{P}, \Phi\rangle$ such that $\Phi$ consists only of facts, we denote with

$$
\Phi^{+}=\left\{a_{1}>\cdots>a_{n} \leftarrow \mid a_{i}>a_{i+1} \leftarrow \in \Phi^{*} \text { for } i \in[1 . . n-1] \wedge n \text { is maximum }\right\}
$$

the set of preference rules which can be obtained from the "merging" of ground preference facts.

Lemma 2 Let $\langle\mathcal{P}, \Phi\rangle$ be a prioritized program such that $\Phi$ consists only of preference facts and $\operatorname{ground}(\Phi)=\Phi^{+}$. Then, $\mathcal{A S O}(\langle\mathcal{P}, \Phi\rangle) \subseteq \mathcal{P} \mathcal{A S}(\langle\mathcal{P}, \Phi\rangle)$. 
Proof. Consider the two graphs $G_{\mathcal{A S O}}=\left(V, E_{\mathcal{A S O}}\right)$ and $G_{\mathcal{P A S}}=\left(V, E_{\mathcal{P A S}}\right) . \quad\left(M_{1}, M_{2}\right) \in$ $E_{\mathcal{P A S}}$ means that $M_{1} \sqsupset M_{2}$, i.e. that

i) there is a ground rule $\varrho_{1}: e_{1}>e_{2} \leftarrow \in \Phi^{*}$ such that $e_{1} \in M_{1}-M_{2}, e_{2} \in M_{2}-M_{1}$, and

ii) there is no ground rule $\varrho_{2}: e_{3}>e_{4} \leftarrow \in \Phi^{*}$, such that $e_{3} \in M_{2}-M_{1}$ and $e_{4} \in M_{1}-M_{2}$.

This implies that if $\operatorname{ground}(\Phi)=\Phi^{+}$

i) there must be a ground rule $\sigma_{1}: \cdots>e_{1}>\cdots>e_{2}>\cdots \leftarrow \in \Phi^{+}$such that $e_{1} \in M_{1}-M_{2}, e_{2} \in M_{2}-M_{1}$, and

ii) there must not be ground rule $\sigma_{2}: \cdots>e_{3}>\cdots>e_{4}>\cdots \leftarrow \in \Phi^{+}$, such that $e_{3} \in M_{2}-M_{1}$ and $e_{4} \in M_{1}-M_{2}$.

Therefore, $M_{1} \sqsupset M_{2}$ also with respect to the $\mathcal{A S O}$ semantics, and the graph $G_{\mathcal{A S O}}$ contains an $\operatorname{arc}\left(M_{1}, M_{2}\right)$. Consequently, as $E_{\mathcal{P} \mathcal{A S}} \subseteq E_{\mathcal{A S O}}, \mathcal{A S O}\left(\left\langle\mathcal{P}, \Phi^{+}\right\rangle\right) \subseteq \mathcal{P} \mathcal{A S}\left(\left\langle\mathcal{P}, \Phi^{+}\right\rangle\right)$.

To find a tight relation between the two semantics, we consider a further restriction of $\Phi^{+}$which is obtained by deleting atoms which do not appear in any model from the ground preference rules:

$$
\begin{aligned}
\widehat{\Phi}^{+}=\left\{a_{1}>\cdots>a_{n} \leftarrow \mid\right. & a_{i}>a_{i+1} \leftarrow \in \Phi^{*} \text { for } i \in[1 . . n-1] \wedge n \text { is maximum } \wedge \\
& \left.\exists M \in \mathcal{S M}(\mathcal{P}) \text { s.t. } a_{i}, a_{i+1} \in M\right\}
\end{aligned}
$$

Theorem 3 Let $\langle\mathcal{P}, \Phi\rangle$ be a prioritized program such that $\Phi$ consists only of preference facts and ground $(\Phi)=\widehat{\Phi}^{+}$. Then, $\mathcal{A S O}(\langle\mathcal{P}, \Phi\rangle)=\mathcal{P} \mathcal{A S}(\langle\mathcal{P}, \Phi\rangle)$.

Proof. $\mathcal{A S O}(\langle\mathcal{P}, \Phi\rangle) \subseteq \mathcal{P} \mathcal{A S}(\langle\mathcal{P}, \Phi\rangle)$ derives from Lemma 2 , as $\widehat{\Phi}^{+}$is derived from $\Phi^{+}$by deleting nodes which do not appear in any model and do not influence the relation $\sqsupset$ in the $\mathcal{A S O}$ semantics.

To show that $\mathcal{A S O}(\langle\mathcal{P}, \Phi\rangle) \supseteq \mathcal{P} \mathcal{A S}(\langle\mathcal{P}, \Phi\rangle)$ consider the relation in the $\mathcal{A S O}$ semantics. $M_{1} \sqsupset M_{2}$ means that

1. there is a ground rule $\sigma_{1}: \cdots>e_{1}>\cdots>e_{2}>\cdots \leftarrow \in \widehat{\Phi}^{+}$such that $e_{1} \in M_{1}-M_{2}$, $e_{2} \in M_{2}-M_{1}$, and

2. there is no ground rule $\sigma_{2}: \cdots>e_{3}>\cdots>e_{4}>\cdots \leftarrow \in \Phi^{+}$, such that $e_{3} \in M_{2}-M_{1}$ and $e_{4} \in M_{1}-M_{2}$.

This implies that if $\operatorname{ground}(\Phi)=\widehat{\Phi}^{+}$

1. there must be a ground rule $\varrho_{1}: e_{1}>e_{2} \leftarrow \in \Phi^{*}$ such that $e_{1} \in M_{1}-M_{2}, e_{2} \in$ $M_{2}-M_{1}$, and

2. there is no ground rule $\varrho_{2}: e_{3}>e_{4} \leftarrow \in \Phi^{*}$, such that $e_{3} \in M_{2}-M_{1}$ and $e_{4} \in M_{1}-M_{2}$. 
Therefore, $M_{1} \sqsupset M_{2}$ also holds with respect to the $\mathcal{P} \mathcal{A S}$ semantics. Consequently, as $E_{\mathcal{A S O}} \subseteq R_{\mathcal{P} \mathcal{A S}}, \mathcal{A S O}\left(\left\langle\mathcal{P}, \Phi^{+}\right\rangle\right) \supseteq \mathcal{P} \mathcal{A S}\left(\left\langle\mathcal{P}, \Phi^{+}\right\rangle\right)$.

An extension of the $\mathcal{A S O}$ semantics has been proposed by Brewka (2004) and Brewka, Niemela, and Truszczynski (2005). In more detail, Brewka (2004) provided a preference description language, allowing to express complex preferences by combining qualitative and quantitative penalty based preferences, whereas Brewka et al. (2005) proposed a framework to specify problem solutions (outcomes) and preferences among them. The latter proposal combines ideas from answer-set programming, answer-set optimization and CPnets (Boutilier, Brafman, Domshlak, Hoos, \& Poole, 2004). The semantics that we have proposed in this paper is different from both those proposed by Brewka (2004) and Brewka et al. (2005), as in some cases it returns different results (see Examples 2 and 4).

\section{Other Approaches}

Besides the approaches managing preferences among atoms, some other works proposed in the literature specify preferences among rules.

Early proposals expressing preferences on rules focus on Default Logic (Brewka \& Eiter, 2000; Delgrande et al., 2000b; Rintanen, 1998), whereas more recently the emphasis has been given to logic programs. In this regard, different proposals have been developed for representing and reasoning about user preferences such as ordered logic programs (Delgrande et al., 2000a; Van Nieuwenborgh \& Vermeir, 2002, 2004) and preferred answer sets of extended logic programs (Brewka \& Eiter, 1999). Most of the approaches propose an extension of Gelfond and Lifschitz's extended logic programming by adding preference information (Delgrande et al., 2003; Wang et al., 2000; Zhang \& Foo, 1997). Other proposals attempt to extend the well founded semantics to logic programs with preferences (Brewka, 1996; Schauba \& Wang, 2001), and an extension of van-Gelder's alternating fixpoint theory for logic programs with priorities has been proposed by Wang et al. (2000).

Gelfond and Son (1997) have proposed a methodology of reasoning with prioritized default in the language of logic programming under answer set semantics. This approach enables the specification of preferences among rules and allows the definition of a set of default rules which must be satisfied as well as a second set of default rules which could be ignored.

Ordered logic programs have been introduced by Delgrande et al. (2000a) as extended logic programs whose rules are subject to a strict partial order with both static and dynamic preferences. This approach is fully prescriptive as it enforces the ordering information during the construction of the answer set. The original program is transformed into a second extended logic program in which preferences are taken into account in the sense that the answer sets obtained by evaluating the transformed theory correspond to the preferred answer sets of the original theory.

Another methodology in which logic programs containing preferences on the set of rules can be translated into logic programs under stable model semantics has been proposed by Delgrande et al. (2003). 


\section{Conclusions}

In this paper the case of preferences involving atoms in logic programming has been studied. In particular, the behavior of the technique proposed by Sakama and Inoue (2000) and Brewka et al. (2003) has been analyzed and a semantics, interpreting each preference rule as a tool for representing a choice over alternative options, has been proposed. Specifically, the proposed approach extends the $\mathcal{P S M}$ semantics by considering a refinement of the natural order among preferences and introduces the concept of comparable models. Preferences and logic programs are examined together in order to determine the choice order and the sets of models which can be compared.

The new semantics has been compared with the $\mathcal{P S M}$ and the $\mathcal{A S O}$ semantics. Complexity analysis has also been performed showing that the use of additional information, regarding the preference order and the sets of non comparable models, does not increase the complexity of computing preferred stable models. Although the semantics presented here has the same complexity as other approaches proposed in the literature, the advantage lies in the fact that it seems to better capture the intuitive meaning of prioritized programs by also considering the structural information of preference rules.

Prioritized reasoning in logic programming under the $\mathcal{P} \mathcal{A S}$ semantics can be easily implemented on the top of deductive systems based on stable model semantics such as DeRes, DLV, Smodels (Cholewinski, Marek, \& Truszczynski, 1996; Leone, Pfeifer, Faber, Calimeri, \& Dell'Armi, 2002; Syrjanen \& Niemela, 2001). An architecture and a system prototype implementing prioritized reasoning (with different semantics) on the top of the DLV system has been presented by Caroprese, Trubitsyna, and Zumpano (2007).

\section{Acknowledgments}

A preliminary version of the papers has been presented by Greco, Trubitsyna, and Zumpano (2006). The authors would like to thank the anonymous referees for their useful suggestions and Filippo Furfaro for his comments.

\section{References}

Boutilier, C., Brafman, R., Domshlak, C., Hoos, H., Poole, D. (2004). CP-nets: A tool for representing and reasoning with conditional ceteris paribus preference statements. Journal of Artificial Intelligence Research, 21, 135-191.

Brewka, G. (1996). Well-Founded Semantics for Extended Logic Programs with Dynamic Preferences. Journal of Artificial Intelligence Research, 4, 19-36.

Brewka, G., Eiter, T. (1999). Preferred Answer Sets for Extended Logic Programs. Artificial Intelligence, 109(1-2), 297-356.

Brewka, G., Eiter, T. (2000). Prioritizing Default Logic. Intellectics and Computational Logic, Kluwer, 27-45.

Brewka, G. (2002). Logic programming with ordered disjunction. Proceedings 18th National Conference on Artificial Intelligence (AAAI/IAAI), 100-105. 
Brewka, G., Niemela, I., Truszczynski, M. (2003). Answer Set Optimization. Proceedings 18th International Joint Conference on Artificial Intelligence (IJCAI), 867-872.

Brewka, G. (2004). Complex Preferences for Answer Set Optimization, Proceedings 9th International Conference on Principles of Knowledge Representation and Reasoning (KR), 213-223.

Brewka, G., Niemela, I., Truszczynski, M. (2005). Prioritized Component Systems. Proceedings 20th National Conference on Artificial Intelligence (AAAI), 596-601.

Caroprese, L., Trubitsyna, I., Zumpano, E. (2007). Implementing Prioritized Reasoning in Logic Programming. Proceedings International Conference on Enterprice Information Systems (ICEIS), 94-100.

Cholewinski, P., Marek, V. W., Truszczynski, M. (1996). Default Reasoning System DeReS. Proceedings 5th International Conference on Principles of Knowledge Representation and Reasoning (KR), 518-528.

Delgrande, J., P., Schaub, T., Tompits, H. (2000). Logic Programs with Compiled Preferences. Proceedings 14th European Conference on Artificial Intelligence (ECAI), 464-468.

Delgrande, J., P., Schaub, T., Tompits, H. (2000). A Compilation of Brewka and Eiter's Approach to Prioritization. Proceedings European Workshop on Logics in Artificial Intelligence (JELIA), 376-390.

Delgrande, J., P., Schaub, T., Tompits, H. (2003). A Framework for Compiling Preferences in Logic Programs. Theory and Practice of Logic Programming, 3(2), 129-187.

Eiter, T., Gottlob, G., Leone, N. (1997). Abduction from Logic Programs: Semantics and Complexity. Theoretical Computer Science 189(1-2), 129-177.

Eiter, T., Gottlob, G., Mannila, H. (1997). Disjunctive Datalog. ACM Transaction On Database Systems, 22(3), 364-418, 1997.

Gelfond, M., Lifschitz, V. (1988). The Stable Model Semantics for Logic Programming, Proceedings International Conference on Logic Programming (ICLP), 1070-1080.

Gelfond, M., Lifschitz, V. (1991). Classical Negation in Logic Programs and Disjunctive Databases, New Generation Computing, 9, 365-385.

Gelfond, M., Son, T.C. (1997). Reasoning with prioritized defaults. Proc. 3rd International Workshop on Logic Programming and Knowledge Representation (LPKR), 164-223.

Greco, S., Trubitsyna, I., Zumpano, E. (2006). On the Semantics of Logic Programs with Preferences. Proceedings 10th European Conference on Logics in Artificial Intelligence (JELIA), 203-215.

Grell, S., Konczak, K., Schaub, T. (2005). nomore<: A System for Computing Preferred Answer Sets. Proceedings 8th International. Conference on Logic Programming and Nonmonotonic Reasoning (LPNMR), 394-398.

Janhunen, T., Niemela, I., Simons, P., You, J.-H. (2000). Unfolding partiality and disjunctions in stable model semantics, Proceedings 7 th International Conference on Principles of Knowledge Representation and Reasoning (KR), 411-419. 
Inoue, K., Sakama, S. (1998). Negation as Failure in the Head. Journal of Logic Programming, 35(1), 39-78.

Kakas, A. C., Kowalski, R. A., Toni, F. (1992). Abductive Logic Programming. Journal of Logic anc Computation, 2(6), 719-770.

Leone, N., Pfeifer, G., Faber, W., Calimeri, F., Dell'Armi, T., Eiter, T., Gottlob, G., Ianni, G., Ielpa, G., Koch, K., Perri, S., Polleres, A. (2002). The DLV System. Proceedings 8th European Conference on Logics in Artificial Intelligence (JELIA), 537-540, 2002.

Minker, J. (1982). On Indefinite Data Bases and the Closed World Assumption, Proc. 6-th Conf. on Automated Deduction, 292-308, 1982.

Papadimitriou, C. H. (1994). Computational Complexity. Addison-Wesley.

Rintanen J. (1998). Complexity of Prioritized Default Logics, Journal of Artificial Intelligence Research, 9, 423-461.

Sakama, C., Inoue, K. (2000). Priorized logic programming and its application to commonsense reasoning. Artificial Intelligence, 123, 185-222.

Schaub, T., Wang , K. (2001). A Comparative Study of Logic Programs with Preference. Proceedings 17th International Joint Conference on Artificial Intelligence (IJCAI), 597-602.

Syrjanen, T., and Niemela, I. (2001). The Smodels System. Proceedings International Conference on Logic Programming and Nonmonotonic Reasoning (LPNMR), 434-438.

Van Nieuwenborgh, D., Vermeir, D. (2002). Preferred Answer Sets for Ordered Logic Programs. Proceedings 10th European Conference on Logics in Artificial Intelligence (JELIA), 432-443.

Van Nieuwenborgh, D., Vermeir, D. (2002). Ordered Diagnosis, Proceedings 10th International Conference on Logic for Programming, Artificial Intelligence, and Reasoning (LPAR), 244-258.

Van Nieuwenborgh, D., Heymans, S., Vermeir, D. (2004). On Programs with Linearly Ordered Multiple Preferences. Proceedings International Conference on Logic Programming (ICLP), 180-194.

Wakaki, T., Inoue, K., Sakama, C., Nitta, K. (2003). Computing Preferred Answer Sets in Answer Set Programming. Proceedings 10th International Conference on Logic for Programming, Artificial Intelligence, and Reasoning (LPAR), 259-273.

Wakaki, T., Inoue, K., Sakama, C., Nitta, K. (2004). The PLP System. Proceedings 9th European Conference on Logics in Artificial Intelligence (JELIA), 706-709.

Wang, K., Zhou, L., Lin, F. (2000). Alternating Fixpoint Theory for Logic Programs with Priority. Proceedings First International Conference on Computational Logic, 164-178.

Zhang, Y., Foo, N. (1997). Answer sets for prioritized logic programs. Proceedings International Logic Programming Symposium (ILPS), 69-83. 